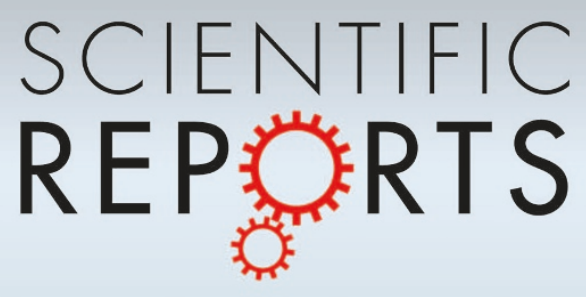

OPEN

SUBJECT AREAS:

DISPLAYS

ELECTRICAL AND ELECTRONIC

ENGINEERING

APPLIED PHYSICS

Received

20 May 2014

Accepted

7 August 2014

Published

22 August 2014

Correspondence and requests for materials should be addressed to H.S. (sasaki@nict.go.jp)

\section{Large size three-dimensional video by electronic holography using multiple spatial light modulators}

\author{
Hisayuki Sasaki, Kenji Yamamoto, Koki Wakunami, Yasuyuki Ichihashi, Ryutaro Oi \& Takanori Senoh
}

Universal Communication Research Institute, National Institute of Information and Communications Technology, 4-2-1 Nukui-kitamachi, Koganei, Tokyo 184-8795, Japan.

In this paper, we propose a new method of using multiple spatial light modulators (SLMs) to increase the size of three-dimensional (3D) images that are displayed using electronic holography. The scalability of images produced by the previous method had an upper limit that was derived from the path length of the image-readout part. We were able to produce larger colour electronic holographic images with a newly devised space-saving image-readout optical system for multiple reflection-type SLMs. This optical system is designed so that the path length of the image-readout part is half that of the previous method. It consists of polarization beam splitters (PBSs), half-wave plates (HWPs), and polarizers. We used $16(4 \times 4)$ $4 \mathrm{~K} \times 2 \mathrm{~K}$-pixel SLMs for displaying holograms. The experimental device we constructed was able to perform $20 \mathrm{fps}$ video reproduction in colour of full-parallax holographic 3D images with a diagonal image size of $85 \mathrm{~mm}$ and a horizontal viewing-zone angle of 5.6 degrees.

T he spatial image reproduction three-dimensional (3D) display methods can display natural 3D images. They can reconstruct spatial images in a similar manner to when objects are being directly observed. These methods can be achieved using integral photography ${ }^{1-3}$ or holography. Integral photography is a method that reproduces discrete light rays from objects. Electronic holography is considered to be the ideal and ultimate $3 \mathrm{D}$ video display method since it can reconstruct the wavefronts of the light reflected by objects and propagated through space. There exist advantages and disadvantages of each method. It is desirable for both the image size of a 3D image and the viewing-zone angle to be large. However, there exist many technical problems to overcome. One of the most important problems remaining to be solved for electronic holography is enlargement of the image size.

A wide range of research has been conducted concerning 3D displays using electronic holography for reproducing 3D videos. This includes research directed towards increasing the image size ${ }^{4-6}$ and research directed towards real-time operation using conventional central processing units $(\mathrm{CPUs})^{7}$ or graphics processing units $(\mathrm{GPUs})^{8-10}$ for hologram calculations.

Various methods for increasing the viewing zone by using space-division multiplexing have been proposed ${ }^{11-13}$. These methods are affected by the problem that part of the viewing-zone angle is missing. To overcome this problem, a method has been proposed that uses a beam splitter and multiple spatial light modulators (SLMs) ${ }^{14}$. However, the viewing-zone angle is scalable only in the horizontal direction, and each of the gaps between SLMs must equal the width of an SLM.

On the other hand, various methods of increasing the image size by using space-division multiplexing have also been proposed. Some problems with these proposals are that they only provide parallax in the horizontal direction $^{15,16}$, the image size is only scalable in the horizontal direction ${ }^{17}$, or high-refresh-rate SLMs are required because the display frame rate is inversely proportional to the space-division multiplexing count ${ }^{18}$.

In a previous paper, we proposed a method of increasing the image size using space-division multiplexing by locating an optical system containing a lens array and other components in front of multiple SLMs. Various methods of colourizing reconstructed images by using space-division multiplexing have been proposed ${ }^{19-22}$. Although these methods achieve ideal colourization of the reconstructed 3D images without colour breakup, they are too complex to adapt to the multiple-SLM system mentioned above because they will require triple the number of SLMs than in a monochromatic system for static colourization and they will also require a large or complex combination of optical systems. We proposed a colourization technique ${ }^{23,24}$ based on time-division 


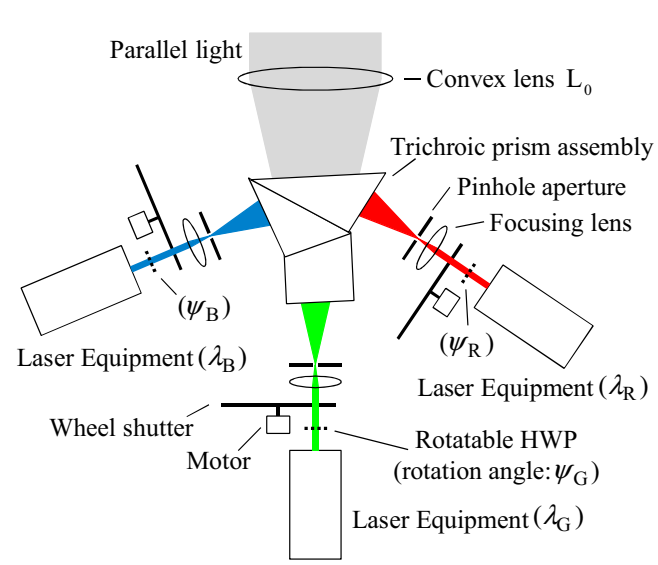

(a)

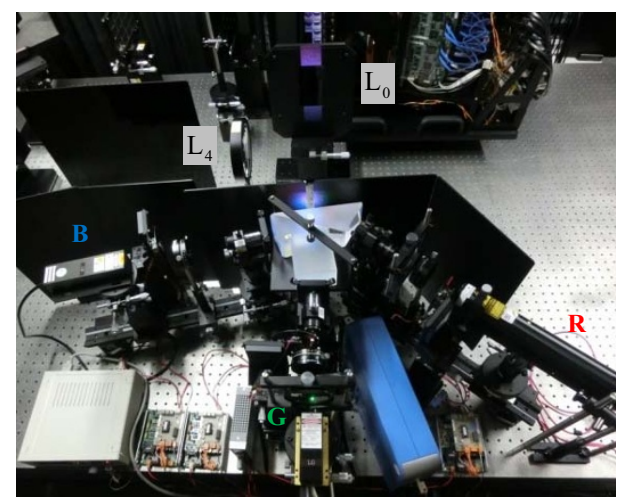

(c)

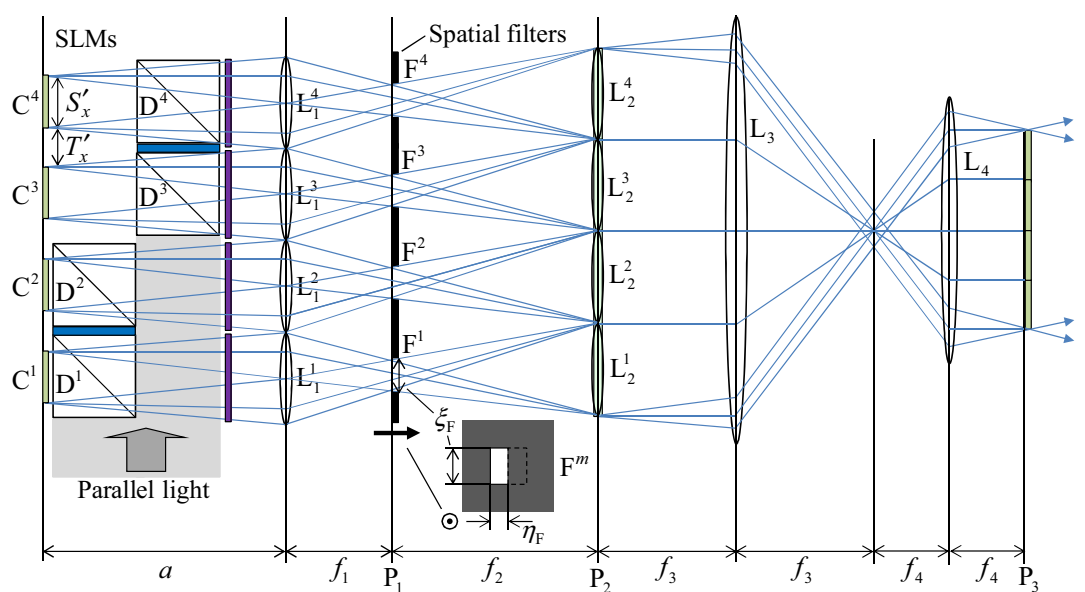

(b)

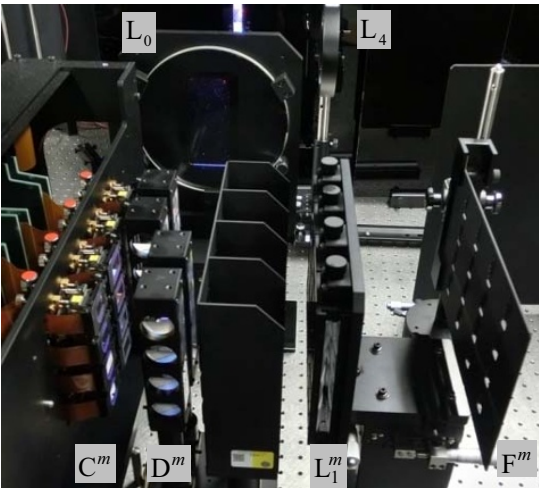

(d)

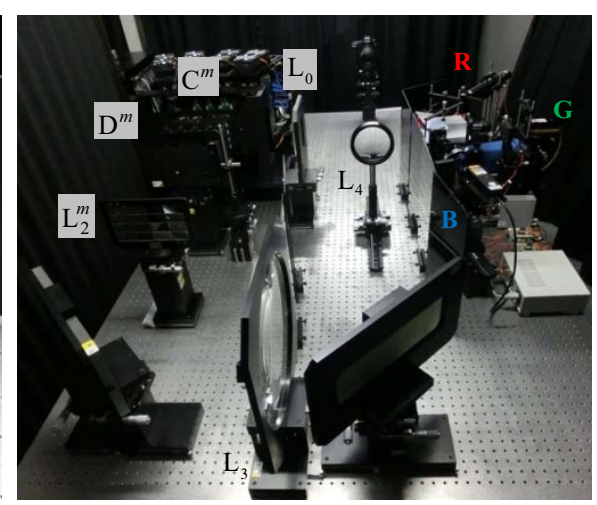

(e)

Figure 1 Configuration of optical systems and their experimental setup. Light source optical system (a) and (c). Image-readout optical system and a part of gap reduction optical system (b) and (d). Overall view of optical systems (e). H.S. took the photographs.

multiplexing ${ }^{25-32}$ using three lasers (i.e., red, green, and blue) and a set of three revolving wheel shutters synchronized with $60 \mathrm{~Hz}$ driven SLMs.

However, a problem with the method mentioned above is the increased size of the image-readout optical system caused by increasing the number of SLMs to obtain a larger image. The enlargement of this assembly in the direction of the SLM's surface is acceptable, but the enlargement in the direction of its optical axis causes a problem. The proposed method can be scaled to increase the number of SLMs for further enlargement of the image, but there exist three factors limiting the image size. Firstly, the sizes of the lenses, secondly, the physical path length between SLMs and lenses to install the imagereadout optical system, and thirdly, vignetting of the first-order diffraction beam by the outer edge of the polarization beam splitter (PBS) that was located at a distance from the SLMs. A solution for the second and third problems is to reduce the number of PBS steps to shorten the path length in the image-readout optical system.

In this paper, we propose a method of producing larger colour electronic holographic images with a newly devised space-saving image-readout optical system for multiple reflection-type SLMs. This optical assembly is designed so that the physical path length of the image-readout part is half that of the previous method and larger number of SLMs can be tiled. We apply this method with 16 (4 $\times 4) 4 \mathrm{~K} \times 2 \mathrm{~K}$-pixel SLMs to display larger full-parallax colour holographic $3 \mathrm{D}$ video than with the previous system ${ }^{23,24}$.

\section{Results}

Limitation of the number of PBS steps in the image-readout optical system. Figure 1 shows the configuration of the developed optical systems and their experimental setup and Table 1 shows a list of parameters. They are examples of the case when the horizontal number of SLMs $K_{x}$ is four. Time-divided colour parallel light from a coherent light source as shown in Figs. 1(a) and 1(c) is incident perpendicularly on SLMs $\mathrm{C}^{m}$ through PBSs $\mathrm{D}^{m}\left(m=1,2, \cdots, K_{x}\right)$ in Figs. 1(b) and 1(d). Note that the superscript of the letter represents the location within the array. Figures $1(\mathrm{a})$ and 1(b) show plane figures of the optical systems in the horizontal direction. Consequently, this is so-called in-line holography. Amplitude modulation (AM) type reflective SLMs $\mathrm{C}^{m}$ are used for displaying hologram data. This kind of SLM creates modulation from the polarization rotation of incident light that corresponds to the input signals. Therefore, the combination of the SLM and each corresponding polarization-selective device (such as a PBS) results in the AM output of the light. The hologram data displayed on $\mathrm{C}^{m}$ are synchronized with the wheel shutters and changed to accommodate the alternating wavelengths of the light sources (i.e., red, green, or blue) every 1/60 second. Since the pixels are arranged in a grid pattern on a thin hologram, the light reflected on $\mathrm{C}^{m}$ contains a primary beam (first-order diffraction beam), conjugate beam (minus first-order diffraction beam), carrier beam (zero-order beam), and high-order diffraction beams of each of them. Since the object beam is generated as a primary beam, the other beams are unnecessary light and must be eliminated.

In the previous system, an image-readout optical system as shown as Fig. 2(a) was used. PBSs are installed in a step-wise manner. We set the physical path length $a$ and the geometrical path length $a^{\prime}$, which was optically compensated by the refractive index of the PBS, between $\mathrm{C}^{m}$ and lenses $\mathrm{L}_{1}^{m}$ as follows: 


$$
a^{\prime}=a+\left(\frac{1}{n_{D}}-1\right) \xi_{D}=\frac{f_{1}^{2}}{f_{2}}+f_{1},
$$

where $n_{\mathrm{D}}$ is the relative refractive index of the PBS material for air. The sizes (width and depth) of the PBSs are $\xi_{\mathrm{D}} \cdot f_{1}$ and $f_{2}$ are the focal lengths of lenses $\mathrm{L}_{1}^{m}$ and $\mathrm{L}_{2}^{m}$, respectively. Equation (1) indicates that $a^{\prime}$ equals the sum of $a$ and the optical effect from the difference of the refractive index caused by a PBS in the optical path. The limitation of the number of PBS steps $l_{\mathrm{D} a}$ that is limited by $a$ is necessary to satisfy the following relation:

$$
a \geq l_{\mathrm{D} a} \xi_{\mathrm{D}}
$$

The thickness of the polarizers and lenses and the difference of $n_{\mathrm{D}}$ according to wavelength play only small or negligible roles.

On the other hand, when the number of SLMs increased, the PBSs in the outer positions produce the vignetting angled part of the readout light from the corresponding SLMs (i.e., light with an angle between the zero- and first-order diffraction beams). To avoid this vignetting by the outermost $\mathrm{PBS}$, limitations of the number of PBS steps $l_{\mathrm{D} x}$ and $l_{\mathrm{D} y}$, which depend on the vignetting limits of the horizontal and vertical directions, respectively, are necessary to satisfy the following relations:

$$
\begin{gathered}
\xi_{\mathrm{D}} \geq 2\left(l_{\mathrm{D} x}+1 / n_{\mathrm{D}}-1\right) \xi_{\mathrm{D}} \tan \theta_{1 x}+S_{x}^{\prime}, \\
\eta_{\mathrm{D}} \geq\left(l_{\mathrm{D} y}+1 / n_{\mathrm{D}}-1\right) \xi_{\mathrm{D}} \tan \theta_{1 y}+S_{y}^{\prime},
\end{gathered}
$$

where the height of the PBSs is $\eta_{\mathrm{D}}$, and the sizes (width and height) of the SLMs are denoted by $S_{x}^{\prime}$ and $S_{y}^{\prime}$. The angles of the first-order diffraction beam $\theta_{1 x}$ and $\theta_{1 y}$ are given as follows:

$$
\begin{aligned}
& \theta_{1 x}=\arcsin \frac{\lambda}{2 p_{x}} \simeq \frac{\lambda}{2 p_{x}}, \\
& \theta_{1 y}=\arcsin \frac{\lambda}{2 p_{y}} \simeq \frac{\lambda}{2 p_{y}},
\end{aligned}
$$

where the horizontal and vertical pixel pitches are $p_{x}$ and $p_{y}$, and the wavelength of the reproduced light is denoted by $\lambda$. Therefore, the overall limitation of the number of PBS steps $l_{\mathrm{D}}$, which depends on either the effect of the physical path length $a$ (derived from $f_{1}$ and $f_{2}$ : cf. formulas (1) and (2)) or vignetting of the first-order diffraction beam by the outermost PBS (cf. formulas (3) or (4)), is given as follows:

$$
\begin{aligned}
l_{D} & =\min \left\{l_{\mathrm{D} a}, l_{\mathrm{D} x}, l_{\mathrm{D} y}\right\}, \\
& =\min \left\{\left\lfloor\frac{a}{\xi_{\mathrm{D}}}\right\rfloor,\left\lfloor\frac{\xi_{\mathrm{D}}-S_{x}^{\prime}}{2 \xi_{\mathrm{D}} \tan \theta_{1 x}}-\frac{1}{n_{\mathrm{D}}}+1\right\rfloor,\left\lfloor\frac{\eta_{\mathrm{D}}-S_{y}^{\prime}}{\xi_{\mathrm{D}} \tan \theta_{1 y}}-\frac{1}{n_{\mathrm{D}}}+1\right\rfloor\right\}^{(7)}
\end{aligned}
$$

where the notation $|\cdot|$ indicates the floor function. $l_{\mathrm{D}}$ is equal to the limitation of the horizontal number of PBSs by the optical system in Fig. 2(a).

When $\eta_{\mathrm{D}}$ equals $\xi_{\mathrm{D}}$, the contribution to $l_{\mathrm{D}}$ by the effect of horizontal direction $l_{\mathrm{D} x}$ is more dominant than the effect of vertical direction $l_{\mathrm{D} y}$ in equation (7) because $S_{x}^{\prime}$ is larger than $S_{y}^{\prime}$ and $\theta_{1 x}$ equals $\theta_{1 y}$ in most cases. Furthermore, $l_{\mathrm{D} a}$, which depends on $a$, has enough margin to install PBSs in a step-wise manner if we set the values of $f_{1}$ and $f_{2}$ appropriately. It is clear from formulas (1) and (2) that we can easy to enlarge $l_{\mathrm{D} a}$ by elongation of $f_{1}$. Therefore, it can be said that most effective parameter in equation (7) is $l_{\mathrm{D} x}$.

The relations between $\xi_{\mathrm{D}}$ and the limitation of the horizontal number of PBSs determined by $l_{\mathrm{D}}$ are drawn as graphs in Fig. 3, where the above-mentioned parameters are selected as the values shown in Table 1. The dark gray shaded area shows feasible numbers of PBS steps. When PBSs with size $\xi_{\mathrm{D}}=\eta_{\mathrm{D}}=28 \mathrm{~mm}$ are used, the upper limit of the number of steps is three and it is clear that we cannot arrange four PBSs with four steps regardless of whether we would like to arrange the SLMs $4 \times 4$ in a plane.

To overcome the limitation of image size scalability, it is necessary to increase the gap $T^{\prime}$ between SLMs and use a large PBS, or to divide the beam from the light source and make it incident from multiple directions (e.g., enter from opposite sides) to reduce the number of PBS steps as shown in Fig. 2(b). However, the first method mentioned in the previous sentence necessitates huge optical components in the following optical system. For example, if we use PBSs with size $\xi_{\mathrm{D}}=35 \mathrm{~mm}$ so that the upper limit of the number of steps is as high as five, the physical sizes of lenses $\mathrm{L}_{2}^{m}$ and $\mathrm{L}_{3}$ (i.e., $\xi_{2}, \eta_{2}$, and $\phi_{3}$ ) would have to be 1.25 times larger than the values shown in Table 1. Furthermore, the second method requires many optical components to create a complex incident light path. These methods are feasible but difficult or complicated.

Therefore, we devised an optical system in which illumination light is enterable from only one direction and the number of PBS steps can be halved. Figures 2(c) and 2(d) show its composition. The limitation of the horizontal number of PBSs by the optical systems in Figs. 2(b)-2(d), which is also drawn as graphs in Fig. 3, is expressed as $2 l_{\mathrm{D}}$ because there exist two PBSs in the horizontal direction for every one step of PBSs in the depth direction. The light and dark gray

\section{Table 1 | System parameters}

Wavelength of lasers $\left(\lambda_{R}, \lambda_{G}, \lambda_{B}\right)$

Number of pixels for each SLM (horizontal $N_{x}^{\prime}$, vertical $N_{y}^{\prime}$ )

Pixel pitch for each SLM (horizontal $p_{x}$, vertical $p_{y}$ )

Image size for each SLM $S^{\prime}$ (horizontal $S_{x}^{\prime}$, vertical $S_{y}^{\prime}$ )

Viewing-zone angle for each SLM (horizontal $\theta_{x}$, vertical $\theta_{y}$ )

Number of SLMs $K$ (horizontal $K_{x}$, vertical $K_{y}$ )

Gap between adjacent SLMs $T^{\prime}$ (horizontal $T_{x}^{\prime}$, vertical $T_{y}^{\prime}$ )

Size of PBS (width and depth $\xi_{\mathrm{D}}$, height $\eta_{\mathrm{D}}$ )

Relative refractive index of $\mathrm{PBS}$ material for air $n_{\mathrm{D}}$

Lens $L_{0}$ (focal length $f_{0}$, diameter $\phi_{0}$ )

Focal lengths of lenses $L_{1}^{m}\left(f_{1}, f_{11}, f_{12}\right)$

Size of lenses $\mathrm{L}_{1}^{m}$ (horizontal $\xi_{1}$, vertical $\eta_{1}$ )

Size of apertures in spatial filters $\mathrm{F}^{m}$ (horizontal $\xi_{\mathrm{F}}$, vertical $\eta_{\mathrm{F}}$ )

Lenses $\mathrm{L}_{2}^{m}$ (focal length $f_{2}$, horizontal size $\xi_{2}$, vertical size $\eta_{2}$ )

Lens $L_{3}$ (focal length $f_{3}$, diameter $\phi_{3}$ )

Lens $L_{4}$ (focal length $f_{4}$, diameter $\phi_{4}$ )

Hologram data update rate

\begin{tabular}{l}
\multicolumn{1}{c}{ Design value } \\
$(633,532,473)[\mathrm{nm}]$ \\
$(3840,2160)$ [pixels] \\
$(4.8,4.8)[\mu \mathrm{m}]$ \\
Diagonal $21.1(18.4,10.4)[\mathrm{mm}]$ \\
$(5.6,2.8)[$ degrees] \\
$16(4,4)$ \\
$(33.2,18.7)[\mathrm{mm}]$ \\
$(28,28)[\mathrm{mm}]$ \\
1.52 \\
$(600,200)[\mathrm{mm}]$ \\
$(125,170,476)[\mathrm{mm}]$ \\
$(45,29)[\mathrm{mm}]$ \\
$(12.4,6.2)[\mathrm{mm}]$ \\
$(350,51.6,29.0)[\mathrm{mm}]$ \\
$(800,340)[\mathrm{mm}]$ \\
$(285,130)[\mathrm{mm}]$ \\
$60[\mathrm{fps}]$
\end{tabular}




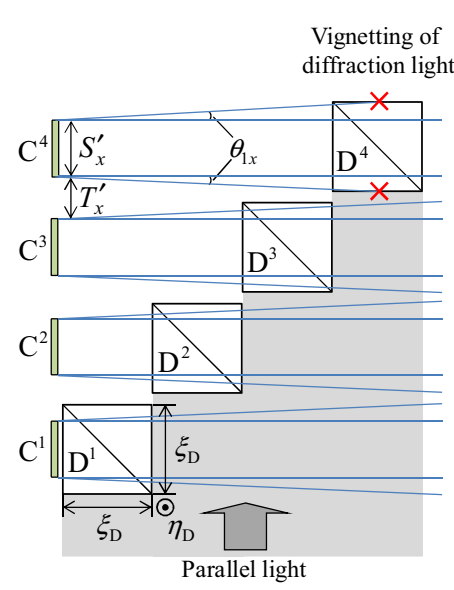

(a)

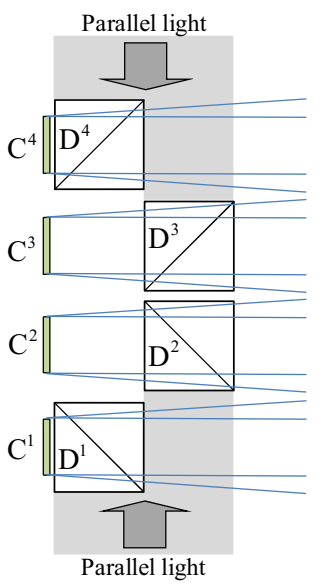

(b)

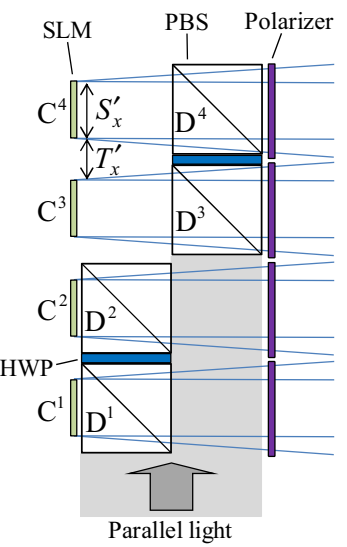

(c)

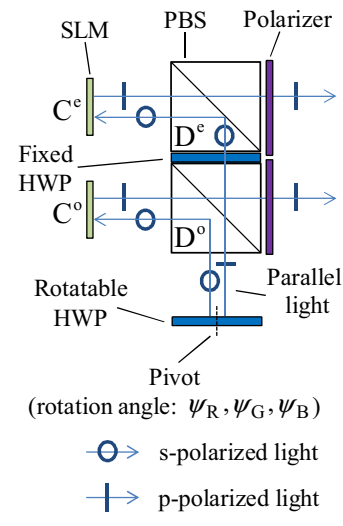

(d)

Figure $2 \mid$ Image-readout optical systems. AM type reflective SLMs without polarization filters are used for displaying hologram data. Combination of the SLM and each corresponding PBS makes AM output of the light. The PBS in the outer position $\mathrm{D}^{4}$ of conventional image-readout optical system produces the vignetting angled part of the readout light from the corresponding SLM C ${ }^{4}$ (a). Illumination light enters from multiple directions (e.g., enters from opposite sides) to reduce the number of PBS steps (b). However, this system requires a complex incident optical system. Newly devised image-readout optical system, which consists of pairs of PBSs, SLMs, HWPs, and polarizers (c), and its operating principles (d). The PBS and SLM at odd-numbered positions are $\mathrm{D}^{\circ}$ and $\mathrm{C}^{\circ}$, and at even-numbered positions are $\mathrm{D}^{\mathrm{e}}$ and $\mathrm{C}^{\mathrm{e}}$. The number of PBS steps can be halved with illumination light entering the PBSs from a single direction.

shaded areas in Fig. 3 show feasible regions of the horizontal number of PBSs in this instance.

Experimental setup. We constructed a device with the parameters shown in Table 1 . We used a combination lens as $\mathrm{L}_{1}^{m}$ to reduce optical distortions. The focal lengths of the individual lenses $\mathrm{L}_{11}^{m}$ and $\mathrm{L}_{12}^{m}$ in $\mathrm{L}_{1}^{m}$ are denoted by $f_{11}$ and $f_{12}$, respectively. This device, which uses an optical system and 16 SLMs, corresponds to an SLM with approximately 133 megapixels. We used AM type $4 \mathrm{~K} \times 2 \mathrm{~K}$-pixel reflective liquid crystal on silicon (LCOS) SLMs ${ }^{33,34}$ that are manufactured by

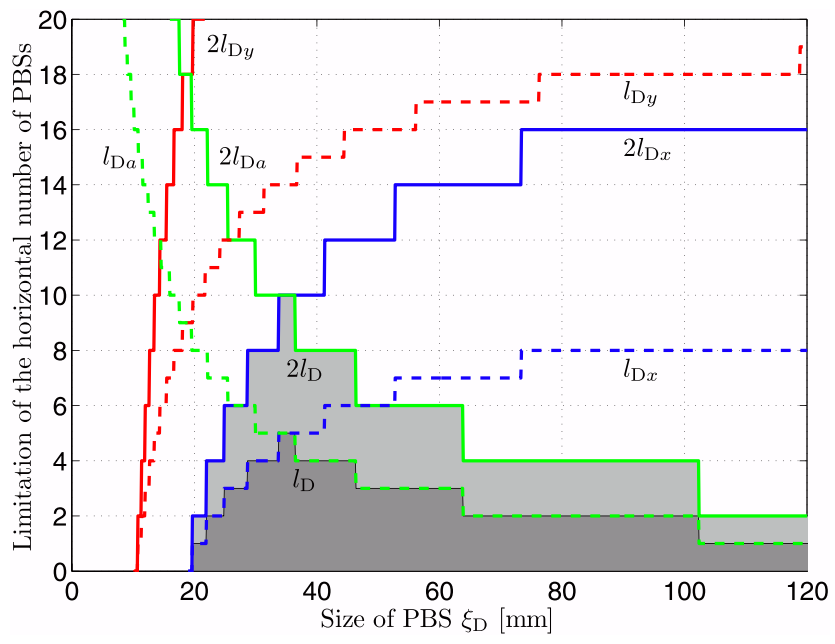

Figure 3 The relations between the size of PBS $\xi_{\mathrm{D}}$ and the limitation of the horizontal number of PBSs $\left(f_{1}=125 \mathrm{~mm}, f_{2}=350 \mathrm{~mm}, n_{\mathrm{D}}=1.52\right.$, $\eta_{\mathrm{D}}=\xi_{\mathrm{D}}, S_{x}^{\prime}=18.4 \mathrm{~mm}, S_{y}^{\prime}=10.4 \mathrm{~mm}, p_{x}=p_{y}=4.8 \mu \mathrm{m}$, and $\lambda=\lambda_{\mathrm{B}}=$ $473 \mathrm{~nm}$ ). The dashed lines show the relations of $\xi_{\mathrm{D}}$ to $l_{\mathrm{D} a}$ (green line), which is the limitation by the path length $a, l_{\mathrm{D} x}$ (blue line), which is the limitation by the vignetting limits of the horizontal direction, $l_{\mathrm{D} y}$ (red line), which is the limitation by the vignetting limits of the vertical direction, and $l_{\mathrm{D}}$ (upper bound of gray shaded area), which is the overall limitation, by the conventional optical system shown in Fig. 2(a). The solid lines show the relations of $\xi_{\mathrm{D}}$ to $2 l_{\mathrm{D} a}, 2 l_{\mathrm{D} s} 2 l_{\mathrm{D} y}$, and $2 l_{\mathrm{D}}$ by the space-saving optical systems shown in Figs. 2(b)-2(d).
JVC KENWOOD Corporation. Figures 1(c)-1(e) show the experimental setup. We performed the following experiments using hologram data created by computer-generated holography (CGH) technology. Note that in this device, the horizontal and vertical gaps between adjacent SLMs $T_{x}^{\prime}$ and $T_{y}^{\prime}$ are 1.8 times the image size of each SLM $S_{x}^{\prime}$ and $S_{y}^{\prime}$ due to physical constraints of the SLMs. In other words, for a display area of 1 for the image part, the area of the missing segments is approximately 4.5 .

Experiment testing image-readout optical system. We operated the rotatable half-wave plates (HWPs), which are installed on light paths from every colour laser (cf. Figs. 1(a) and 2(d)), to control the ratio of the s- and p-polarized light that is incident on the PBSs. We defined the rotation angles of the HWPs $\psi_{\mathrm{R}}, \psi_{\mathrm{G}}$, and $\psi_{\mathrm{B}}$ to be 0 degrees when the illuminated SLMs of $\mathrm{C}^{\mathrm{e}}$ are brightest and the illuminated SLMs of $\mathrm{C}^{\circ}$ are darkest. When the HWPs are rotated until $\mathrm{C}^{\mathrm{e}}$ and $\mathrm{C}^{\circ}$ have equivalent brightness, $\psi_{\mathrm{R}}, \psi_{\mathrm{G}}$, and $\psi_{\mathrm{B}}$ become 24,20 , and 23 degrees, respectively. Furthermore, when they are rotated until the $\mathrm{C}^{\mathrm{e}}$ are darkest and $\mathrm{C}^{\circ}$ are brightest, $\psi_{\mathrm{R}}, \psi_{\mathrm{G}}$, and $\psi_{\mathrm{B}}$ become 45,44 , and 45 degrees, respectively. Figure 4 shows the experimental results in each colour channel. From these results, we confirmed that the devised optical system is able to illuminate the SLMs from a single direction, the number of PBS steps can be halved, and the intended function of the image-readout is achieved.

Experiment displaying 3D images. We confirmed that objects with different depths could be reconstructed in colour by using timedivision multiplexing. Figures 5 and 6 show colour 3D images that are displayed by this system. Figures 5(a) and 6(a) show the design values of the displayed objects. The displayed hologram data was calculated by using the ray-sampling (RS) plane approach ${ }^{35}$. In this approach, the RS plane is virtually set near the object and the dense ray information is sampled and converted into the wavefront on that plane as the Ray-Wavefront conversion process ${ }^{36}$. The light field on the hologram plane is then obtained by a two-dimensional (2D) light propagation calculation from the given RS plane to the hologram plane. The dense ray information was rendered as a set of projection images by general computer-graphics (CG) software.

The photographs in Figs. 5(b), 5(c), and 5(d) were taken from a location directly in front, a location determined by pointing 2.8 degrees right, and a location obtained by pointing 2.8 degrees left, 


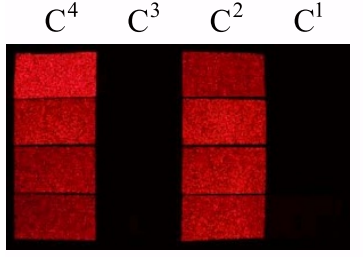

$\psi_{\mathrm{R}}=0^{\circ}$

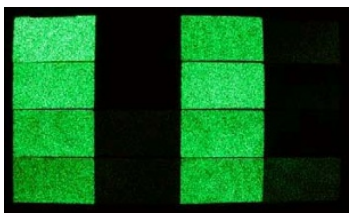

$\psi_{\mathrm{G}}=0^{\circ}$

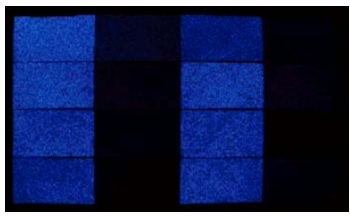

$\psi_{\mathrm{B}}=0^{\circ}$

(a)

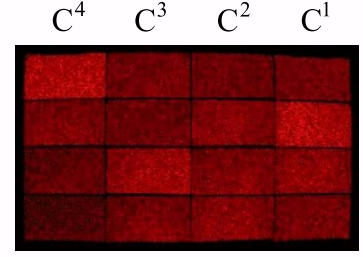

$\psi_{\mathrm{R}}=24^{\circ}$

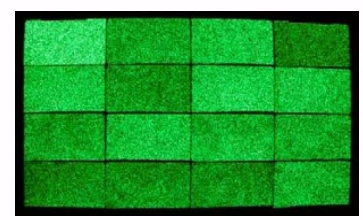

$\psi_{\mathrm{G}}=20^{\circ}$

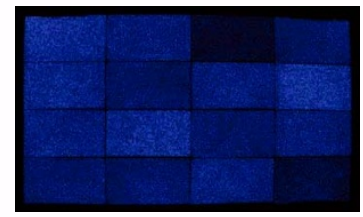

$\psi_{\mathrm{B}}=23^{\circ}$

(b)

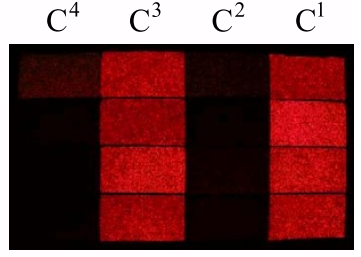

$\psi_{\mathrm{R}}=45^{\circ}$

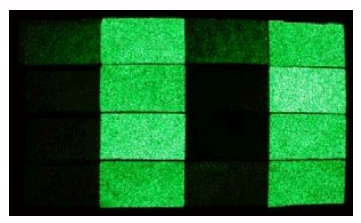

$\psi_{\mathrm{G}}=44^{\circ}$

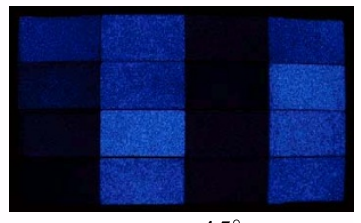

$\psi_{\mathrm{B}}=45^{\circ}$

(c)

Figure 4 Control of incident light distributions using rotatable HWPs in each colour channel. The illuminated SLMs of $\mathrm{C}^{\mathrm{e}}\left(\mathrm{i}\right.$.e., $\mathrm{C}^{2}$ and $\mathrm{C}^{4}$ ) are brightest and the illuminated SLMs of $\mathrm{C}^{\circ}$ (i.e., $\mathrm{C}^{1}$ and $\mathrm{C}^{3}$ ) are darkest and the rotation angles $\psi_{\mathrm{R}}, \psi_{\mathrm{G}}$, and $\psi_{\mathrm{B}}$ of the HWPs are defined as 0 degrees (a); the HWPs are rotated until $\mathrm{C}^{\mathrm{e}}$ and $\mathrm{C}^{\circ}$ have equivalent brightness (b); and they are rotated until the $\mathrm{C}^{\mathrm{e}}$ are darkest and $\mathrm{C}^{\circ}$ are brightest $(\mathrm{c})$.

respectively. A logotype of NICT is located at $113 \mathrm{~mm}$, the left front wheel of a car is at $118 \mathrm{~mm}$, the left rear wheel is at $143 \mathrm{~mm}$, and a Venus statue is at $120 \mathrm{~mm}$ behind the hologram plane, respectively.

The photograph in Fig. 6(b) was taken of the reconstructed objects focused on the hologram plane, the photographs in Figs. 6(c)-6(e) were focused on the logotype of NICT, and the photographs in Figs. 6(f)-6(h) were focused on the background object. Figs. 6(b), 6(d), and 6(g) were taken from locations directly in front; Figs. $6(\mathrm{c})$ and $6(\mathrm{f})$ were taken by pointing 2.8 degrees right; and Figs. 6(e) and 6(h) were taken by pointing 2.8 degrees left. The logotype of NICT is located at $100 \mathrm{~mm}$, and the back-

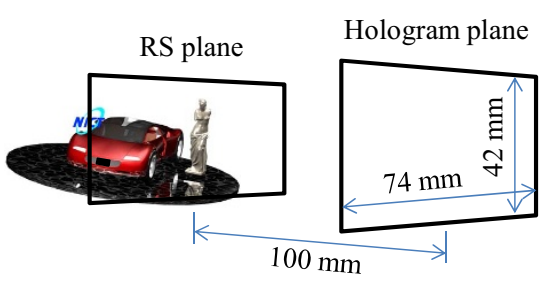

(a)

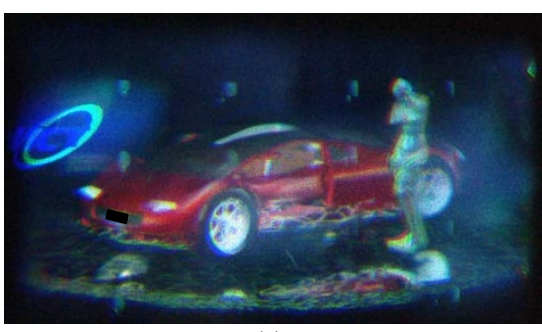

(c) ground object is $200 \mathrm{~mm}$ behind the hologram plane. When the objects can be reconstructed at different depths, the background object should be blurry in Figs. 6(c)-6(e), and the logotype of NICT should be blurry in Figs. 6(f)-6(h). From the experimental results and the description above, it is apparent that objects with different depths can be reconstructed.

The photographs in Figs. 5 and 6 also show the views of the same displayed objects from other angles. It is apparent that the reconstructed objects can be observed in the designed viewing zone and missing segments that can be seen without the optical system are eliminated.

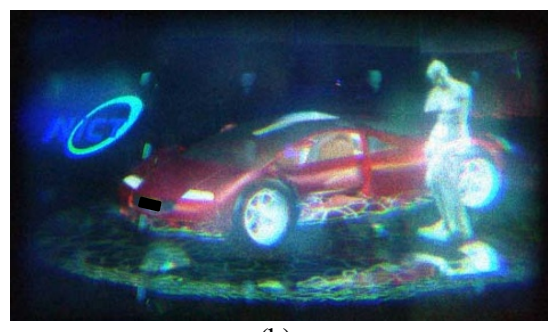

(b)

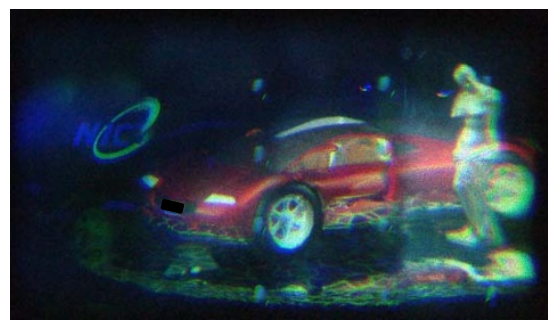

(d)

Figure 5 Experimental results. Design values of the displayed objects in the experiment (a). A logotype of NICT is located at $113 \mathrm{~mm}$, the left front wheel of a car is at $118 \mathrm{~mm}$, the left rear wheel is at $143 \mathrm{~mm}$, and a Venus statue is at $120 \mathrm{~mm}$ behind the hologram plane, respectively. Photographs that were taken from locations directly in front (b), taken by pointing 2.8 degrees right (c), and 2.8 degrees left (d). K.W. prepared hologram data. H.S. took the photographs. 


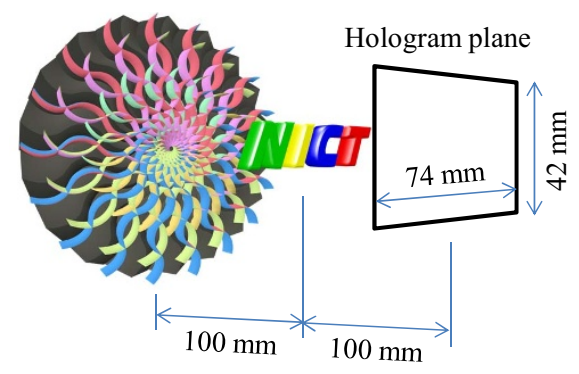

(a)

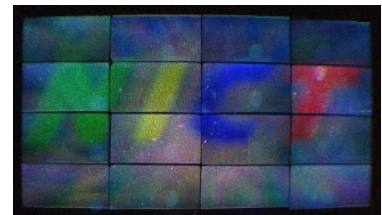

(b)

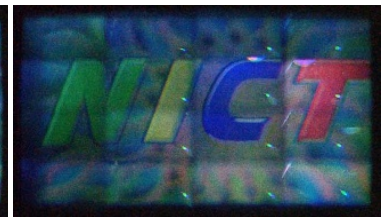

(e)

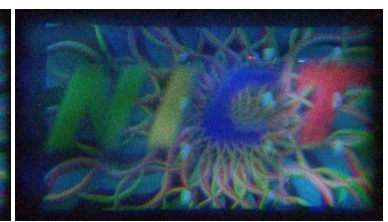

(h)

Figure $6 \mid$ Experimental results. Design values of the displayed objects (a). Photographs that were taken of the reconstructed objects focused on the hologram plane (b), focused on the logotype of NICT (c)-(e), and focused on the background object (f)-(h). Photographs (b), (d), and (g) were taken from locations directly in front; (c) and (f) were taken by pointing 2.8 degrees right; and (e) and (h) were taken by pointing 2.8 degrees left. K.W. prepared hologram data. H.S. took the photographs.

We also confirmed that a video could be reproduced. Figure 7 shows the experimental results. We used CGH technology to create hologram data for the earth and letters. The letters circle around the earth. The logotype of NICT is located at $104 \mathrm{~mm}$, and the centre of the earth is $127 \mathrm{~mm}$ behind the hologram plane. This experiment confirmed that this video sequence could be converted to a frame rate with one set of red, green, and blue per frame in time-division colour and played smoothly at a $20 \mathrm{fps}$ (frames per second).

The displayed size of these 3D images is $74 \mathrm{~mm} \times 42 \mathrm{~mm}$ (i.e., the diagonal is $85 \mathrm{~mm}$ ) and the viewing-zone angles are 5.6 degrees horizontally and 2.8 degrees vertically. From the experiments described above, it is apparent that a colour-moving image of $3 \mathrm{D}$ objects having an image size of 16 times that of an SLM can be played with a frame rate of 20 fps using the proposed method.

Figures 5-7 show that gaps between the SLMs are eliminated. However, the boundaries of the SLMs are observed. In addition, differences of brightness are observed between each small display area.

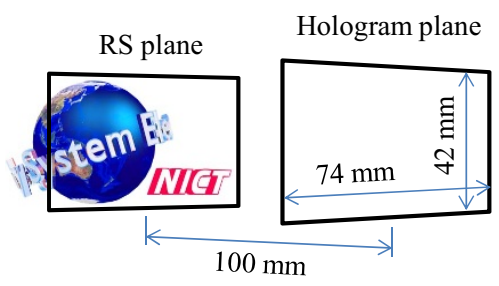

(a)

\section{Discussion}

In this paper, we propose a method of producing larger colour electronic holographic images with a newly devised space-saving imagereadout optical system for multiple reflection-type SLMs. We applied the proposed method to 16 SLMs to realize full-parallax video holography at a frame rate of $20 \mathrm{fps}$ with an image size of 16 times that of one SLM and a viewing-zone angle of 5.6 degrees. Although the timedivision colourization method had a frame rate of 20 fps because we used 60 -fps SLMs, we could also reach $60 \mathrm{fps}$ in colour, for example, if 180 -fps SLMs were used.

When observing a 3D image with the focus on a distant location from the hologram plane in the depth direction, the boundaries of the SLMs become diffuse and sparse because these boundaries exist on the hologram plane. Actually, the observed boundaries are sparser in Figs. 6(f)-6(h), which are focused on the background object located far from the hologram plane, than the boundaries in Figs. 6(c)-6(e), which are focused on the logotype of NICT located near the hologram plane.

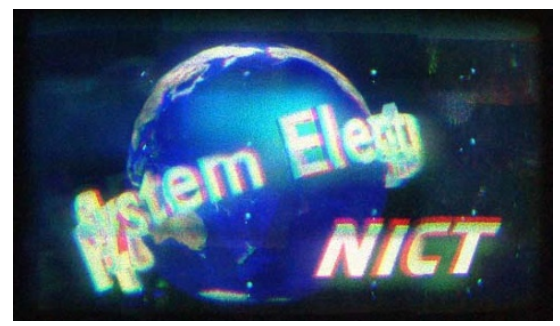

(b)

Figure $7 \mid$ Experimental results. Design values of the displayed objects (a) and reconstructed 3D image at video rate (b). The centre of the earth is $27 \mathrm{~mm}$ behind the RS plane. K.W. prepared hologram data. H.S. took the photograph. 
Differences of brightness exist depending on the difference of contrast between each display area in Figs. 4-7. Since there is no difference of contrast among SLMs, we think that differences among the optical specifications of the various PBSs that comprise an incident optical system become the dominant influence. Since each PBS provides a different contrast, this produces a detrimental effect for viewing the $3 \mathrm{D}$ image. We believe that we can balance the differences of display brightness among the various display areas if we compensate for luminance differences among the hologram data that is displayed on the various SLMs.

\section{Methods}

Space-saving image-readout optical system. Figures 2(c) and 2(d) show the spacesaving image-readout optical system and its operating principles. Rotatable HWPs are inserted in the light paths from every colour laser because light in which s- and ppolarizations are mixed with the intended ratio is to be incident on the PBSs. We use pairs of PBSs. We denote a PBS and SLM at odd-numbered positions here by $\mathrm{D}^{\circ}$ and $\mathrm{C}^{\mathrm{o}}$ and at even-numbered positions by $\mathrm{D}^{\mathrm{e}}$ and $\mathrm{C}^{\mathrm{e}}$ in a pair of PBSs and their corresponding SLMs, respectively. $\mathrm{D}^{\circ}$ and $\mathrm{D}^{\mathrm{e}}$ are arranged in tandem for the incident light path, and a HWP is fixed between these PBSs. The s-polarized component of the incident light is reflected by the mirror (e.g., dielectric multilayer mirror, wire grid, etc.) in $\mathrm{D}^{\circ}$ and illuminates $\mathrm{C}^{\circ}$. The p-polarized component passes through $\mathrm{D}^{\circ}$ and is converted to s-polarized light by the HWP. This light is incident on and reflected by $D^{\mathrm{e}}$ so that it then illuminates $\mathrm{C}^{\mathrm{e}}$.

Readout light from each SLM consists of mixed polarization light because the spolarized incident light changed to the p-polarized component of the readout light, and this conversion ratio depends on the displayed value of each pixel. Only the ppolarized component of the readout light passes through the PBS and polarizer and is then output from this image-readout optical system. The s-polarized component from the SLMs is reflected by the PBSs and goes back towards the light source. The light that goes back to $\mathrm{D}^{\circ}$ from $\mathrm{D}^{\mathrm{e}}$ is also converted to p-polarized light by the HWP and goes straight through $\mathrm{D}^{\circ}$ ideally. However, there exists a possibility of crosstalk interference by a small quantity of a residual s-polarized component. The polarizer next to $\mathrm{D}^{\circ}$ cuts off this s-polarized component. Essentially, a polarizer does not have to be set next to $\mathrm{D}^{\mathrm{e}}$, but we use it for luminance equalization of the $\mathrm{D}^{\circ}$ and $\mathrm{D}^{\mathrm{e}}$ optical outputs. Since the modulation type of the SLMs is AM as mentioned previously, polarizers after the PBSs will not affect the modulated wavefront of holograms.

The loss of light that passes through $\mathrm{D}^{\mathrm{e}}$ is greater than the loss of light that only passes through $\mathrm{D}^{\circ}$ because it takes a longer path and also has to pass through the HWP. There exists a possibility of incomplete conversion in the HWP due to wavelength differences, which also leads to a loss of light in $\mathrm{D}^{\mathrm{e}}$. To compensate for these effects, rotatable HWPs are installed in the light paths from every colour laser as mentioned above. These HWPs can be rotated to control the ratio of the s- and pcomponents of the incident light and to compensate for luminance differences in the $\mathrm{D}^{\circ}$ and $\mathrm{D}^{\mathrm{e}}$ optical outputs of every colour. Essentially, rotatable HWPs do not have to be set if the corresponding laser equipment is rotatable around each optical axis and fixable at intended attitudes, but we use them to facilitate adjustments of polarizations. The optical systems in Fig. 1 utilize this method.

Image size and viewing-zone angle. If we denote the number of SLM pixels here by $N_{x}$ and $N_{y}$ and the pixel pitch in the horizontal and vertical directions by $p_{x}$ and $p_{y}$, respectively, then the image sizes $S_{x}$ and $S_{y}$ and the horizontal and vertical viewingzone angles $\theta_{x}$ and $\theta_{y}$ are given as follows:

$$
\begin{gathered}
S_{x}=N_{x} p_{x}, \\
S_{y}=N_{y} p_{y}, \\
\theta_{x}=2 \theta_{1 x}, \\
\theta_{y}=\theta_{1 y},
\end{gathered}
$$

where the horizontal and vertical angles of the first-order diffraction beam $\theta_{1 x}$ and $\theta_{1 y}$ are given by equations (5) and (6). With the space-division multiplexing method, if we denote the number of pixels in the horizontal and vertical directions of each SLM by $N_{x}^{\prime}$ and $N_{y}^{\prime}$ and denote the number of SLMs by $K_{x}$ and $K_{y}$, then $N_{x}=N_{x}^{\prime} K_{x}$ and $N_{y}=N_{y}^{\prime} K_{y}$. The only difference between the horizontal and vertical directions is the viewing-zone angles $\theta_{x}$ and $\theta_{y}$.

Light source optical system and colourization. Figure 1(a) shows the light source optical system that implements colourization based on time-division multiplexing ${ }^{23,24}$. This is also known as the frame sequential method. This system consists of three lasers (i.e., red, green, and blue), rotatable HWPs, wheel shutters, spatial filters, and a trichroic prism assembly. The shutters are rotating wheels driven by motors that are synchronized with the SLMs and controlled so that a beam of one of the three colours is selected to pass through the shutter every $1 / 60$ second. After that, the laser beams pass through a spatial filter (i.e., convex lens and pinhole) so that the shape of the beams gradually becomes wider. Three beams are combined into one by a Phillips type trichroic prism assembly. Finally, the combined white beam is collimated by a large size convex lens $\mathrm{L}_{0}$ to illuminate all of the SLMs.

Gap reduction optical system. Figure 1(b) shows the image-readout and gap reduction optical systems. Emitted light from the image-readout optical system is incident on lenses $\mathrm{L}_{1}^{m}$, spatial filters $\mathrm{F}^{m}$, and lenses $\mathrm{L}_{2}^{m 6}$. Spatial filter $\mathrm{F}^{m}$ is located on the common focal plane $\mathrm{P}_{1}$ of lenses $\mathrm{L}_{1}^{m}$ and $\mathrm{L}_{2}^{m}$, and the focal length $f_{1}$ of $\mathrm{L}_{1}^{m}$ and focal length $f_{2}$ of $\mathrm{L}_{2}^{m}$ are related as follows:

$$
f_{2}=\left(\frac{S^{\prime}+T^{\prime}}{S^{\prime}}\right) f_{1},
$$

depending on the image size $S^{\prime}$ of each SLM and the gap $T^{\prime}$ between SLMs. Also, we set the physical path length $a$ and the geometrical path length $a^{\prime}$, which was optically compensated by the refractive index of the PBS, between $\mathrm{C}^{m}$ and lenses $\mathrm{L}_{1}^{m}$ as shown in equation (1). This enlarges the image due to $C^{m}$ by $\left(S^{\prime}+T^{\prime}\right) / S^{\prime}$ and shifts it to $\mathrm{L}_{2}^{m}\left(\mathrm{P}_{2}\right)$.

The missing image segments due to the gaps between the SLMs will disappear. The group of lenses $\mathrm{L}_{1}^{m}$ and $\mathrm{L}_{2}^{m}$ forms the lens arrays, respectively. $\mathrm{F}^{m}$ is a spatial filter array for eliminating the conjugate beam, carrier beam, and high-order beams, which are unnecessary light ${ }^{37}$. Finally, the light is incident on lens $\mathrm{L}_{3}$ and lens $\mathrm{L}_{4}$. By setting the focal length $f_{3}$ of $\mathrm{L}_{3}$ and focal length $f_{4}$ of $\mathrm{L}_{4}$ as follows:

$$
f_{4}=\left(\frac{S^{\prime}}{S^{\prime}+T^{\prime}}\right) f_{3},
$$

the image that had been enlarged at $\mathrm{P}_{2}$ will be returned to its original size at plane $\mathrm{P}_{3}$. This optical system can exhibit parallax in both the horizontal and vertical directions. However, as shown in equations (10) and (11), the horizontal viewing-zone angle $\theta_{x}$ is two times the viewingzone angle of the first-order diffraction beam $\theta_{1 x}$, but the vertical viewing-zone angle $\theta_{y}$ equals $\theta_{1 y}$. The reason for this is that spatial filters $\mathrm{F}^{m}$ eliminate the first-order diffraction beam in the lower side of the vertical direction to eliminate the conjugate image, i.e., single-sideband holography ${ }^{38}$ with half-zoneplate processing is performed for the hologram data that is displayed on the SLM $^{39}$ We used spatial filters $\mathrm{F}^{m}$ as shown in Fig. 1(b). A mask (gray square with white rectangle aperture) shows a plane figure of the configuration of the spatial filter in the vertical direction. The aperture sizes in horizontal and vertical directions of spatial filters $\xi_{\mathrm{F}}$ and $\eta_{\mathrm{F}}$ are given as follows:

$$
\begin{gathered}
\xi_{\mathrm{F}}=2 f_{1} \tan \theta_{1 x}, \\
\eta_{\mathrm{F}}=f_{1} \tan \theta_{1 y} .
\end{gathered}
$$

The conjugate beam and carrier beam are eliminated by the dashed lined rectangle part in the masked area, and the other masked area eliminates the high-order beams. Only the object beam passes through the aperture selectively.

1. Koike, T., Oikawa, M., Utsugi, K., Kobayashi, M. \& Yamasaki, M. Autostereoscopic display with 60 ray directions using LCD with optimized color filter layout. Proc. SPIE 6490, Stereoscopic Displays and Virtual Reality Systems XIV, 64900T, San Jose, CA, USA. doi:10.1117/12.704633. (2007, March 5).

2. Sakai, H., Yamasaki, M., Koike, T., Oikawa, M. \& Kobayashi, M. 41.2: Autostereoscopic display based on enhanced integral photography using overlaid multiple projectors. SID Symposium Digest of Technical Papers 40, 611-614. doi:10.1889/1.3256853. (2009, July 5).

3. Arai, J. et al. Integral three-dimensional television using a 33-megapixel imaging system. J. Disp. Technol. 6, 422-430 (2010).

4. Peyghambarian, N., Tay, S., Blanche, P.-A., Norwood, R. \& Yamamoto, M. Rewritable holographic 3D displays. Opt. Photon. News 19, 22-27 (2008).

5. Blanche, P.-A. et al. Holographic three-dimensional telepresence using large-area photorefractive polymer. Nature 468, 80-83 (2010).

6. Yamamoto, K., Ichihashi, Y., Senoh, T., Oi, R. \& Kurita, T. 3D objects enlargement technique using an optical system and multiple SLMs for electronic holography. Opt. Express 20, 21137-21144 (2012).

7. Yamamoto, K., Mishina, T., Oi, R., Senoh, T. \& Kurita, T. Real-time color holography system for live scene using 4K2K video system. Proc. SPIE 7619, Practical Holography XXIV: Materials and Applications, 761906, San Francisco, CA, USA. doi:10.1117/12.840589. (2010, February 10).

8. Bove, Jr, V. M., Plesniak, W. J., Quentmeyer, T. \& Barabas, J. Realtime holographic video images with commodity PC hardware. Proc. SPIE 5664, Stereoscopic Displays and Virtual Reality Systems XII, 255-262, San Jose, CA, USA. doi:10.1117/12.585888. (2005, June 14).

9. Shiraki, A. et al. Simplified electroholographic color reconstruction system using graphics processing unit and liquid crystal display projector. Opt. Express 17, 16038-16045 (2009).

10. Ichihashi, Y., Oi, R., Senoh, T., Yamamoto, K. \& Kurita, T. Real-time capture and reconstruction system with multiple GPUs for a 3D live scene by a generation from 4K IP images to 8K holograms. Opt. Express 20, 21645-21655 (2012).

11. Mishina, T., Okui, M., Doi, K. \& Okano, F. Holographic display with enlarged viewing-zone using high-resolution LC panel. Proc. SPIE 5005, Practical 
Holography XVII and Holographic Materials IX, 137-144, Santa Clara, CA, USA. doi:10.1117/12.473818. (2003, May 30).

12. Hahn, J., Kim, H., Lim, Y., Park, G. \& Lee, B. Wide viewing angle dynamic holographic stereogram with a curved array of spatial light modulators. Opt. Express 16, 12372-12386 (2008).

13. Chen, R.-Y. \& Wilkinson, T. Field of view expansion for 3-D holographic display using a single spatial light modulator with scanning reconstruction light. $3 D T V$ Conference: The True Vision - Capture, Transmission and Display of 3D Video (3DTV-CON), 2009, 1-4, Potsdam, Germany. doi:10.1109/3DTV.2009.5069673. (2009, May 4-6)

14. Yaraş, F., Kang, H. \& Onural, L. Circular holographic video display system. Opt. Express 19, 9147-9156 (2011).

15. Fukaya, N. et al. Expansion of the image size and viewing zone in holographic display using liquid crystal devices. Proc. SPIE 2406, Practical Holography IX, 283-289. San Jose, CA, USA. doi:10.1117/12.206229. (1995, April 12).

16. Takaki, Y. \& Nakamura, J. Development of a holographic display module using a $4 \mathrm{k} 2 \mathrm{k}$-SLM based on the resolution redistribution technique. OSA Technical Digest: Biomedical Optics and 3D Imaging, DM2C.5, Miami, FL, USA. doi:10.1364/DH.2012.DM2C.5. (2012, April 28 - May 2).

17. Finke, G., Kujawińska, M., Zaperty, W. \& Kozacki, T. Spatiotemporal multiplexing method for big images observation in wide angle holographic display. 3DTV-Conference: The True Vision - Capture, Transmission and Dispaly of 3D Video (3DTV-CON), 2013, 1-4, Aberdeen, UK. doi:10.1109/ 3DTV.2013.6676642. (2013, October 7-8).

18. Slinger, C., Cameron, C. \& Stanley, M. Computer-generated holography as a generic display technology. Computer 38, 46-53 (2005).

19. St-Hilaire, P., Benton, S. A., Lucente, M. E. \& Hubel, P. M. Color images with the MIT holographic video display. Proc. SPIE 1667, Practical Holography VI, 73-84, San Jose, CA, USA. doi:10.1117/12.59642. (1992, May 1).

20. Yaraş, F., Kang, H. \& Onural, L. Real-time color holographic video display system. 3DTV Conference: The True Vision - Capture, Transmission and Display of 3D Video (3DTV-CON), 2009, 1-4, Potsdam, Germany. doi:10.1109/ 3DTV.2009.5069660. (2009, May 4-6).

21. Yaras, F., Kang, H. \& Onural, L. Real-time phase-only color holographic video display system using LED illumination. Appl. Opt. 48, H48-H53 (2009).

22. Senoh, T. et al. Full-color wide viewing-zone-angle electronic holography system. OSA Techinal Digest: Digital Holography and Three-Dimensional Imaging, DTuA3, Tokyo, Japan. doi:10.1364/DH.2011.DTuA3. (2011, May 9-11).

23. Sasaki, H., Yamamoto, K., Ichihashi, Y. \& Senoh, T. Colorization technique for 3D objects enlargement type electronic holography using an optical system and multiple SLMs. OSA Techinal Digest: Digital Holography and Three-Dimensional Imaging, DTh2A.4, Kohala Coast, HI, USA. doi:10.1364/DH.2013.DTh2A.4. (2013, April 21-25).

24. Sasaki, H., Yamamoto, K., Ichihashi, Y. \& Senoh, T. Image size scalable fullparallax coloured three-dimensional video by electronic holography. Sci. Rep. 4, 4000 (2014).

25. Senoh, T., Mishina, T., Yamamoto, K., Oi, R. \& Kurita, T. Wide viewing-zoneangle full-color electronic holography system using very high resolution liquid crystal display panels. Proc. SPIE 7957, Practical Holography XXV: Materials and Applications, 795709, San Francisco, CA, USA. doi:10.1117/12.876763. (2011, February 07 )

26. Shimobaba, T. \& Ito, T. A color holographic reconstruction system by time division multiplexing with reference lights of laser. Optical Review 10, 339-341 (2003).

27. Shimobaba, T., Shiraki, A., Masuda, N. \& Ito, T. An electroholographic colour reconstruction by time division switching of reference lights. J. Opt. A: Pure Appl. Opt. 9, 757 (2007).

28. Shimobaba, T., Shiraki, A., Ichihashi, Y., Masuda, N. \& Ito, T. Interactive color electroholography using the FPGA technology and time division switching method. IEICE Electronics Express 5, 271-277 (2008).

29. Oikawa, M. et al. Time-division color electroholography using one-chip RGB LED and synchronizing controller. Opt. Express 19, 12008-12013 (2011).
30. Martínez, J. L., Martínez-García, A. \& Moreno, I. Wavelength-compensated color Fourier diffractive optical elements using a ferroelectric liquid crystal on silicon display and a color-filter wheel. Appl. Opt. 48, 911-918 (2009).

31. Häussler, R. et al. Large real-time holographic displays: from prototypes to a consumer product. Proc. SPIE 7237, Stereoscopic Displays and Applications XX, 72370S, San Jose, CA, USA. doi:10.1117/12.805873. (2009, February 17).

32. Makowski, M. Minimized speckle noise in lens-less holographic projection by pixel separation. Opt. Express 21, 29205-29216 (2013).

33. Bleha, W. P. \& Sterling, R. D. D-ILA technology for high-resolution projection displays. Proc. SPIE 5080, Cockpit Displays X, 239-249, Orlando, FL, USA doi:10.1117/12.497532. (2003, September 12).

34. Suehiro, K. et al. Integral 3D TV using ultrahigh-definition D-ILA device. Proc. SPIE 6803, Stereoscopic Displays and Applications XIX, 680318, San Jose, CA, USA. doi:10.1117/12.766892. (2008, February 29).

35. Wakunami, K. \& Yamaguchi, M. Calculation for computer generated hologram using ray-sampling plane. Opt. Express 19, 9086-9101 (2011).

36. Wakunami, K., Yamashita, H. \& Yamaguchi, M. Occlusion culling for computer generated hologram based on ray-wavefront conversion. Opt. Express 21, 21811-21822 (2013).

37. Yamamoto, K., Oi, R., Mishina, T. \& Okui, M. Half-zone-plate processing for objects on both sides of hologram display. Proc. SPIE 6912, Practical Holography XXII: Materials and Applications, 69120Q, San Jose, CA, USA. doi:10.1117/ 12.762903. (2008, January 25).

38. Bryngdahl, O. \& Lohmann, A. Single-Sideband Holography. J. Opt. Soc. Am. 58, 620-624 (1968).

39. Mishina, T., Okano, F. \& Yuyama, I. Time-alternating method based on singlesideband holography with half-zone-plate processing for the enlargement of viewing zones. Appl. Opt. 38, 3703-3713 (1999).

\section{Acknowledgments}

The authors sincerely thank Ms. Mao Makino at NICT, Kyoto, Japan. She gave us insightfu comments and suggestions and furnished 3D CG models that were used in experiment shown in Fig. 6.

\section{Author contributions}

H.S. designed the optical systems, did experimental work, wrote the main manuscript text, and prepared figures and videos. K.Y. designed the optical systems, did project planning and management. K.W. prepared hologram data and did experimental work. Y.I., R.O. and T.S. did experimental work. All authors reviewed the manuscript.

\section{Additional information}

Supplementary information accompanies this paper at http://www.nature.com/ scientificreports

Competing financial interests A patent application with priority date 23 April 2013 has been filed for the space-saving image-readout optical system

How to cite this article: Sasaki, H. et al. Large size three-dimensional video by electronic holography using multiple spatial light modulators. Sci. Rep. 4, 6177; DOI:10.1038/ srep06177 (2014).

This work is licensed under a Creative Commons Attribution-NonCommercialShareAlike 4.0 International License. The images or other third party material in this article are included in the article's Creative Commons license, unless indicated otherwise in the credit line; if the material is not included under the Creative Commons license, users will need to obtain permission from the license holder in order to reproduce the material. To view a copy of this license, visit http:// creativecommons.org/licenses/by-nc-sa/4.0/ 